Revue internationale P.M.E.

Économie et gestion de la petite et moyenne entreprise

\title{
Les motivations des entrepreneurs : une étude empirique de 2278 fondateurs d'entreprises dans 14 pays
}

\section{Roger A. Blais et Jean-Marie Toulouse}

Volume 3, numéro 3-4, 1990

URI : https://id.erudit.org/iderudit/1007982ar

DOI : https://doi.org/10.7202/1007982ar

Aller au sommaire du numéro

\section{Éditeur(s)}

Presses de l’Université du Québec

ISSN

0776-5436 (imprimé)

1918-9699 (numérique)

Découvrir la revue

Citer cet article

Blais, R. A. \& Toulouse, J.-M. (1990). Les motivations des entrepreneurs : une étude empirique de 2278 fondateurs d'entreprises dans 14 pays. Revue internationale P.M.E., 3(3-4), 269-300. https://doi.org/10.7202/1007982ar

\section{Résumé de l'article}

Cette étude examine les réponses fournies par 2278 entrepreneurs de 14 pays sur les motifs qui les ont incités à fonder une entreprise. Une première analyse statistique a révélé des divergences appréciables dans la façon dont les entrepreneurs répondaient aux 38 questions sur la motivation et, beaucoup plus important, dans la façon avec laquelle certaines nationalités appréciaient l'importance des motivations. Pour corriger ces modes de réponse, les données originales furent recodées et reclassées en utilisant la méthode statistique de l'équation personnelle. La classification hiérarchique ascendante des données recodées a révélé que les motivations se regroupaient en trois grandes entités régionales : le bloc anglosaxon, le bloc Scandinave, et le bloc mixte qui se compose surtout de pays en voie de développement. Suite à une analyse $\mathrm{f}$ actorielle Varimax par rotation des données recodées et reclassées, sept grands facteurs de motivation se dégagent : besoin de reconnaissance sociale, besoin de développement personnel, besoin d'argent, besoin d'indépendance et d'autonomie, altruisme communautaire, besoin d'évasion, et opportunisme. La distribution de cesfacteurs et des variables qui les constituent montre que les motivations entrepreneuriales transgressent les frontières nationales et transcendent les systèmes culturels des pays. A la lumière de ces résultats, il apparaît assez clairement que la motivation à devenir entrepreneur dans un pays donné est avant tout déterminée par des facteurs métaculturels qui transcendent l'environnement socio-économique immédiat.
Ce document est protégé par la loi sur le droit d'auteur. L'utilisation des services d'Érudit (y compris la reproduction) est assujettie à sa politique d'utilisation que vous pouvez consulter en ligne.

https://apropos.erudit.org/fr/usagers/politique-dutilisation/ 


\title{
Les motivations des entrepreneurs : une étude empirique de 2278 fondateurs d'entreprises dans 14 pays *
}

\author{
Roger A. BLAIS** \\ École Polytechnique de Montréal \\ Jean-Marie TOULOUSE \\ École des H.E.C., Montréal
}

* Une première version de cette étude a été présentée au $35^{\circ}$ congrès mondial du Conseil international de la petite entreprise à Washington en juin 1990. Cette recherche a été rendue possible grâce à l'aide financière du CRSH du Canada, du fonds FCAR du gouvernement du Québec et du MICT du Canada, et à la collaboration des chercheurs dans 14 pays constituant le réseau SARIE de recherche internationale en entrepreneurship dirigé par Sari Scheinberg et par le professeur Ian C. MacMillan du Wharton Business School aux États-Unis. Les auteurs désirent également remercier leurs assistants de recherche, Serge Trépanier, Marc Jarry et Shelina Tajdin, ainsi que le professeur Bernard Clément du Département de mathématiques appliquées de l'École Polytechnique.

** Roger A. Blais, Ph.D., est ingénieur et professeur titulaire à l'École Polytechnique de Montréal où il a été le premier directeur de la recherche, puis responsable des liaisons avec l'industrie. Il a fondé le Centre d'innovation industrielle de Montréal. Son enseignement et ses recherches portent sur l'innovation et l'entrepreneuriat technologique. Il est membre de l'Académie des sciences du Canada, de l'Académie canadienne du génie, Officier de l'Ordre du Canada et auteur d'une centaine de publications, dont cinq livres sur divers sujets scientifiques, sur l'entrepreneuriat et l'innovation technologique. Adresse : Département de génie industriel, Ecole Polytechnique, C.P. 6079, Montréal, Québec, H3C 3A7.

*** Jean-Marie Toulouse, Ph.D. (psychologie sociale), est titulaire de la Chaire d'entrepreneuriat Maclean Hunter de l'École des Hautes Études Commerciales de Montréal où il a été directeur de la recherche, directeur du doctorat et directeur du Département de l'administration des ressources humaines. Ses recherches et publications portent sur l'entrepreneuriat, la stratégie d'entreprise et le travail des gestionnaires. Adresse : Chaire d'entrepreneuriat Maclean Hunter, École des H.E.C., 5255 avenue Decelles, Montréal, Québec, H3T 1 V6. 


\title{
RÉSUME
}

Cette étude examine les réponses foumies par 2278 entrepreneurs de 14 pays sur les motifs qui les ont incités à fonder une entreprise. Une première analyse statistique a révélé des divergences appréciables dans la façon dont les entrepreneurs répondaient aux 38 questions sur la motivation et, beaucoup plus important, dans la façon avec laquelle certaines nationalités appréciaient limportance des motivations. Pour corriger ces modes de réponse, les données originales furent recodées et reclassées en utilisant la méthode statistique de l'équation personnelle. La classification hiérarchique ascendante des données recodées a révélé que les motivations se regroupaient en trois grandes entités régionales : le bloc anglosaxon, le bloc scandinave, et le bloc mixte quise compose surtout de pays en voie de développement. Suite à une analyse factorielle Varimax par rotation des données recodées et reclassées, sept grands facteurs de motivation se dégagent : besoin de reconnaissance sociale, besoin de développement personnel, besoin d'argent, besoin d'indépendance et d'autonomie, altruisme communautaire, besoin d'évasion, et opportunisme. La distribution de cesfacteurs et des variables quiles constituent montre que les motivations entrepreneuriales transgressent les frontières nationales et transcendent les systèmes culturels des pays. A la lumière de ces résultats, il apparait assez clairement que la motivation à devenir entrepreneur dans un pays donné est avant tout déterminée par des facteurs métaculturels qui transcendent l'environnement socio-économique immédiat.

\begin{abstract}
This research bears on the answers provided by 2278 entrepreneurs in 14 countries about their motivation to establish a new enterprise. Preliminary statistical analysis revealed important differences in the way entrepreneurs answer motivation questions (38 of them) and, even more important, in the way certain nationalities gauge the relative importance of motivation factors. To correct these answering modes, the original data were recoded and reclassified according to the personal equation statistical method. Ascending hierarchical classification of the recoded data reveals that motivations are grouped according to three broad regional entities : the Anglo-Saxon Block, the Scandinavian Block, and the Mixed Block which comprises developing countries for the most part. Seven main motivation factors emerge from the rotated Varimax factor analysis of the recoded and reclassified data : need for social recognition, need for selfdevelopment, need for money, need for independence and autonomy, communitarianism, need for escape, and opportunism. The distribution of these factors and of the the underlying variables suggests clearly that entrepreneurial motivations cut across national boundaries and transcend cultural systems of countries. The results indicate that the motivation to become an entrepreneur in a given country is primarily determined by metacultural factors that transcend the immediate socio-economic environment.
\end{abstract}

\section{RESUMEN}

Este estudio esta fundado en las respuestas de 2278 empresarios en 14 paises sobre su motivacion para establecer una nueva empresa. El analisis estadistico ha revelado apreciables divergencias en la forma en la que los empresarios respondieron a las 38 preguntas de motivacion y mucho mas importante, la forma en la que ciertas nacionalidades mostraban la relativa importancia de las motiva-ciones. Para corregir estas formas de respuesta, los datos originales fueron codados y clasificados de nuevo, utilizando el método estadistico de la ecuacion personal. El ascendente analisis jerarquico de los datos recodados ha revelado que las motivaciones se reagrupaban en tres grandes entidades regionales : el bloque anglosajon, el bloque escandinavo, y el bloque mixto constituido esencialmente de paises en via de desarrollo. Tras un analisis factorial Varimax por rotacion de los datos recodados y reclasificados se desprende : necesidad de reconocimiento social, necesidad de desarrollo personal, necesidad de dinero, necesidad de independencia y autonomia, altruismo comunitario, necesidad de evasion, yoportunismo. La distribucion de estos factores y de las variables que los constituyen muestra que las motivaciones empresariales traspasan las fronteras nacionales y transcienden los sistemas culturales de los paises. A la luz de estos resultados, parece bastante claro que la motivacion para ser emprendedor un pais es sobre todo determinado por factores metaculturales que transcienden el medio socio-economico proximo. 


\section{Introduction}

L'activité entrepreneuriale suscite de plus en plus d'intérêt dans diverses régions du monde. En effet, les petites entreprises jouent un rôle important dans la vie économique, tant au niveau de la valeur ajoutée et de la création des nouveaux emplois que du développement régional. Par surcroît, elles interviennent beaucoup dans l'invention et l'exploitation de technologies porteuses de progrès économique. Sur le plan social proprement dit, elles procurent des formes alternatives de développement et fournissent à la société davantage de flexibilité et de souplesse devant les changements et bouleversements de toutes sortes qui agitent le système productif, notamment en matière de nouvelles technologies. Bien plus, elles permettent aux individus de mieux s'exprimer, de s'actualiser, de réaliser leurs ambitions. Mais qui sont ces gens qui créent des entreprises ? Quelles sont leurs motivations? Les entrepreneurs de divers pays se ressemblent-ils ?

\section{Cadre d'étude}

Cette recherche a été entreprise dans le cadre du projet international amorcé en 1985 par Sari Scheinberg sous la direction du professeur Ian C. MacMillan, alors directeur du Centre de développement de l'entreprise à l'Université de New York. Le projet a été réalisé par un réseau international de chercheurs appelé SARIE ( SCheinbergNetwork of AffiliatedResearchersonInternational Entrepreneurship»), dont la coordination pour le Canada fut assurée par R.A. Blais. Les travaux de cueillette et d'analyse des données furent réalisés entre 1986 et 1988.

Les publications résultant de ce projet sont, à ce jour, surtout axées sur la question des motivations, quoique l'équipe canadienne se soit aussi penchée sur la question des valeurs culturelles des entrepreneurs et des non-entrepreneurs.

Au niveau des comparaisons internationales des facteurs de motivation, citons les études de Scheinberg et MacMillan (1988), Alänge et Scheinberg (1988), Blais et Toulouse (1990), Blais, Toulouse et Clément (1990). D'intéressantes études nationales ont été publiées par Alänge et Scheinberg (1988) pour la Suède; par Dubini (1989) pour l'Italie; par Fleming (1988) pour l'Australie; par Blais et al. (1988), Blatt et al. (1989), Kyle et al. (1989), Blais et Toulouse (1989) pour le Canada ainsi que pour le Québec. Toutes les études incitent à examiner de plus près les différences de motivation entre les entrepreneurs de divers pays.

L'originalité de la présente étude réside, d'une part dans la méthodologie de traitement statistique utilisée et, d'autre part, dans la problématique comparative des motivations des entrepreneurs dans autant de pays différents. 


\section{Cadre conceptuel}

Puisqu'il existe déjà une littérature volumineuse sur les motivations des entrepreneurs, nous n'aborderons ici que les éléments qui nous paraissent essentiels. De la même façon, l'espace réservé à cet article nous empêche de faire une analyse de la littérature sur les comparaisons internationales déjà publiées, notamment sur les dirigeants d'entreprises.

Soulignons néanmoins que dès le début des recherches sur l'entrepreneuriat, les auteurs se sont interrogés sur les différences entre les entrepreneurs de divers pays.L'observation initiale suggérait des différences de comportement entrepreneurial d'un pays à l'autre, laissant ainsi croire que les entrepreneurs sont «nationalement» différents. Dans le même sens, on s'est demandé si dans le cadre des sociétés différentes, les personnes se destinant à l'entrepreneuriat seraient semblables. La réponse à ces interrogations a amené les chercheurs à se regrouper sous deux modèles : le premier propose que les entrepreneurs sont semblables dans tous les pays et le second propose le contraire.

\subsection{Tous les entrepreneurs sont semblables}

Pour illustrer cette argumentation, nous nous limiterons à deux auteurs : McClelland pour l'approche individuelle et Hagen pour l'approche collective. Dans son fameux ouvrage "The Achieving Society», McClelland (1961) laisse penser que la dynamique de l'entrepreneuriat est la même dans tous les pays. On peut résumer son point de vue de la façon suivante :

\begin{tabular}{|l}
$\begin{array}{l}\text { les valeurs } \\
\text { et l'idéologie } \\
\text { d'une société }\end{array} \rightarrow \begin{array}{l}\text { les pratiques de } \\
\text { socialisation } \\
\text { dans la famille }\end{array} \rightarrow \begin{array}{l}\text { le besoin d'ac- } \\
\text { complissement } \\
\text { («need for } \\
\text { achievement») }\end{array} \rightarrow \begin{array}{l}\text { le comportement } \\
\text { entrepreneurial }\end{array}$ \\
\hline
\end{tabular}

Pour McClelland (1971), les entrepreneurs auraient les mêmes caractéristiques, les mêmes motivations d'un pays à l'autre.

Cette conception centrée sur les besoins des individus ne permet pas de comprendre la dimension collective et sociale des gestes entrepreneuriaux. Le modèle proposé par Hagen $(1962,1971)$ est ici plus utile. Après avoir étudié plusieurs collectivités, tant en Asie qu'en Amérique du Sud, il constate que l'entrepreneuriat se retrouve chez des gens qui ont été privés de statut par la société dans laquelle ils vivent. Cette privation crée de l'anxiété et de la colère qui se transmettent d'une génération à l'autre. Ces frustrations mènent au désir de changer de situation en devenant créateur, en fondant une nouvelle entreprise. On peut donc penser, en s'inspirant de Hagen, que les entrepreneurs de divers pays auraient un profil différent, mais que leurs motivations fondamentales seraient les mêmes. 


\subsection{Les entrepreneurs sont différents d'une culture à l'autre}

Cochran $(1960,1971)$ offre une explication différente. D'emblée, il propose que les entrepreneurs de l'Amérique latine sont différents des entrepreneurs américains. Il soutient que l'entrepreneur latino-américain aime la théorie, la discussion et le débat; il recherche un statut mais un statut personnel et non résultant d'une activité d'affaires. Ses gestes sont guidés par une quête de dignité personnelle en se référant à ce qui est important pour lui. Dans son étude, Cochran (1971) reconnaît clairement que des conditions culturelles différentes sont susceptibles de produire des entrepreneurs différents parce que les motivations et les pratiques de socialisation des enfants sont différentes au départ.

\subsection{Différences culturelles, différences dans les motivations}

Ces perspectives ont trouvé leur écho dans des études plus récentes, dont celle de Leff (1979) qui démontre que les intérêts, les motivations des entrepreneurs dans les pays moins développés ne sont pas les mêmes que celles que l'on observe dans les pays industrialisés. La différence provient de ce que les mécanismes de fonctionnement des marchés ne sont pas les mêmes : dans les pays développés. Ces mécanismes sont bien établis alors que dans les pays moins développés, ils sont souvent informels et difficiles à anticiper.

Les études sur la création d'entreprises par les immigrants accordent beaucoup d'importance aux différences entre les entrepreneurs. Certains chercheurs (Bonacich, 1973; Pyong Gap Min, 1984; Ward et Jenkins, 1984; Light, 1984; Burgess, 1984) se sont demandés pourquoi les immigrants originaires de l'Inde, de la Turquie ou d'Israël créaient souvent des entreprises alors que ceux originaires du Sud Sahara en créaient plus rarement. Dans le même sens, les études sur la création d'entreprises par les Coréens aux États-Unis ont suscité plusieurs interrogations en référence aux autres groupes ethniques (Pyong Gap Min, 1984, 1987).

Pour d'autres chercheurs, dont Woodrun (1985) et Stewart (1987), les motivations pour créer une entreprise sont fortement influencées par la culture, la valeur et les idéologies de la société d'origine. Ce résultat suggère que dans les divers pays, la motivation pour créer une entreprise n'est pas la même. Ces études n'ont cependant pas encore réussi à démontrer la nature des différences; on se contente de proposer le modèle.

L'objet de la présente étude est justement d'explorer cette question : si l'on admet que la culture influence la motivation des entrepreneurs, il faut donc s'attendre à ce que dans les pays ayant une culture différente, les motivations des entrepreneurs soient différentes.

\subsection{La problématique des motivations entrepreneuriales}

Dans les innombrables études sur l'entrepreneuriat, la question des motivations occupe une place dominante. En effet, depuis longtemps les chercheurs 
s'intéressent à ce qui peut pousser quelqu'un à créer une entreprise. Les études peuvent être regroupées en deux catégories :

(1) Celles qui s'appuient sur la théorie de la motivation, avec McClelland (1961) comme chef de file.

(2) Celles qui explorent les diverses raisons invoquées par les entrepreneurs euxmêmes, comme il en sera fait état ici.

La théorie de la motivation fait appel à la psychologie, aux sciences du management et même à l'anthropologie. Les travaux de McClelland (1961), un psychologue, sont ceux qui ont le plus influencé cette approche. Selon cet auteur, les individus fortement motivés par le désir de réussite («achievement») font preuve de plus de débrouillardise et d'initiative que les autres; ils recherchent continuellement dans leur milieu les occasions de relever des défis intéressants et de se prouver à euxmêmes qu'ils peuvent réussir.

De là à transposer la théorie de McClelland au cas des entrepreneurs, il n'y avait qu'un pas car ces derniers font très souvent preuve d'un N-ACH élevé. Les travaux de Hornady et Aboud (1971) et de Sexton et Bowman (1983) ont renforcé et diffusé largement cette théorie.

Pour McClelland et Winter (1969), les individus sont largement motivés par le désir du pouvoir, de la réussite ou de l'affiliation, qui apparaissent comme autant de variables indépendantes. Pour eux, les entrepreneurs peuvent être fortement motivés par le désir de réaliser une idée ou de se prouver à eux-mêmes qu'ils sont capables de créer une nouvelle entreprise et d'en faire un succès.

Le thème de l'accomplissement, fondé sur les théories de McClelland $(1961,1969)$ et de Maslow (1954), exprime un besoin de réalisation personnelle. Ce thème a été repris par de nombreux auteurs, dont Gasse (1982).

D'autres chercheurs, comme Herzberg, Mausner et Snyderman (1959) ainsi que Collins, Moore et Unwalla (1955), se sont particulièrement intéressés aux motivations mais sous l'angle des réponses fournies par les entrepreneurs euxmêmes. Les raisons invoquées par ces derniers se regroupent sous trois thèmes :

(1) Indépendance : créer son entreprise pour être son propre patron, pour être indépendant, pour diriger plutôt que d'être dirigé, pour fixer son propre horaire de travail, pour engager qui nous plait, et ainsi de suite.

(2) Évasion : créer son entreprise pour se sortir du chômage ou pour éviter des conditions de travail non sécuritaires ou peu hygiéniques, ou pour se libérer d'un patron intransigeant. Il s'agit là de conditions de survie, car il n'y a pas d'autre choix.

(3) Accomplissement : créer une entreprise pour réaliser une nouvelle idée, pour se prouver à soi-même qu'on peut faire un succès d'une nouvelle entreprise, pour mieux mettre à profit ses connaissances et ses talents, pour continuer d'apprendre et de se développer, pour réaliser une ambition, un rêve. 
A l'appui des thèses ci-dessus, Herzberg, Mausner et Snyderman (1959) expliquent que la seule façon de motiver un employé est de lui procurer un travail vraiment stimulant, de lui confier des responsabilités et de lui faire savoir l'estime qu'a son patron pour elle ou pour lui. Une telle «hygiène» du travail s'avère très utile. Son absence rendra le travailleur malheureux et sera souvent la cause du départ d'employés de valeur. Par contre, son existence n'incitera pas nécessairement les employés à travailler plus fort.

De nombreux chercheurs ont développé la thèse du désir d'indépendance, d'autonomie. Par exemple, Collins, Moore et Unwalla (1955) ont amplement montré que l'entrepreneur avait besoin d'avoir le plein contrôle de sa vie au travail, d'être son propre patron. Rotter (1964) parle en termes de «internal locus of control» alors que Hersch et Shiebe (1967) ont montré que les individus ainsi motivés sont des gens qui s'actualisent plus que les autres, qui sont indépendants, efficaces et industrieux. Kent (1982) est également d'avis que l'entrepreneur éprouve de forts besoins d'accomplissement, d'autonomie, d'indépendance et de leadership. Richman (1982) faitéchoaux mêmespropos: pour lui, la motivation principale del'entrepreneur est d'avoir la chance d'exercer des choix libres sur son mode de vie et d'être indépendant, autonome. D'aprèslui, les caractéristiques entrepreneurialesapparaissent très souvent à l'âge de l'adolescence ou de jeune adulte. Enfin, Jacobowitz et Vilder (1982) ont trouvé que leurs sujets entrepreneuriaux possédaient un fort désir d'indépendance, une forte propension à vouloir contrôler leur vie et un sens d'importante responsabilité personnelle dans la prise de décision.

Le désir d'évasion a été suggéré par Shapero (1975) pour de nombreux entrepreneurs. Ces derniers, dit-il, se trouvent expulsés d'une niche familière et confortable et éprouvent la nécessité de prendre un nouveau tournant dans leur vie. Ce thème a été repris par Knight (1983) au Canada qui identifie sept types de «réfugiés entrepreneuriaux». Cette notion de déplacement s'appuie, entre autres, sur les études de Collins, Moore et Unwalla (1955) et de Cooper et Komives (1970).

Rappelons que la question des motivations a aussi été abordée à partir du contexte. Ainsi, O'Reilly et Roberts (1973) et surtout Hofstede (1980) ont montré que le cadre de référence des individus conditionne la nature et l'importance de leurs besoins, de sorte que la culture qui prévaut dans un pays ou même dans une région va affecter les comportements entrepreneuriaux et les modes de gestion des entreprises. Par exemple, les Basques en Espagne et les Canadiens-Français au Canada sont desentités culturelles distinctes, avec descomportements entrepreneuriaux spécifiques (Blais et Toulouse, 1989).

Ces résultats suggèrent donc d'étudier, comme nous le faisons ici, les motivations des entrepreneurs de divers pays. C'est ainsi que nous découvrirons l'importance relative de motivations selon les cultures, par exemple le désir d'aider ses proches ou son groupe ethnique, celui d'atteindre un certain statut social pour soi ou pour sa famille, ou encore de devenir riche. 


\section{Devis de l'étude}

Le devis de recherche ayant déjà été défini par Scheinberg et MacMillan (1988), de même que le fondement théorique du projet, il ne nous reste qu'à rappeler le cadre général d'enquête.

\subsection{Le questionnaire}

L'instrument principal de cueillette de données fut un questionnaire conçu en 1985 par Sari Scheinberg et quelques collègues sur la foi des contributions théoriques de Baumol (1985), Bruno et Tyebjee (1982), Friberg (1985), Hofstede (1980), Lodge (1976), Maslow (1954), McClelland (1961), McClelland et Winters (1969), Shapero et Sokol (1982), et autres chercheurs.

Le questionnaire, traduit en dix langues, comprenait 598 éléments répartis en cinq sections :

1) MOTIVATIONS : 38 éléments, cotés de 1 à 5

(i.e. très important à pas important).

2) VALEURS CULTURELLES : 166 éléments, cotés de 1 à 5 (idem).

3) IDÉOLOGIES : 44 éléments, cotés «oui» ou «non».

4) ENVIRONNEMENT ENTREPRENEURIAL : 194 éléments.

5) DONNÉES PERSONNELLES ET CARACTÉRISTIQUES DE LA FIRME : 156 éléments.

Pour les non-entrepreneurs, les parties 2 et 3 étaient identiques alors que les parties 1 et 5 étaient adaptées et la partie 4 retranchée, donnant lieu en tout à 378 variables ${ }^{1}$.

\subsection{La population étudiée}

Tel qu'indiqué au tableau 1, la population étudiée comprend 2278 entrepreneurs répartis dans 14 pays, de même que 1733 non-entrepreneurs (surtout des gérants de banque, des enseignants et des employés d' entreprises manufacturières) dans neuf de ces mêmes pays. Pour les entrepreneurs, il s'agit de fondateurs d'entreprises créées depuis 1980 et comptant au moins un employé à temps complet.

Sauf pour Porto Rico et le Portugal, où l'enquête fut faite par entrevue téléphonique, toutes les données ont été obtenues par questionnaire mis à la poste. A l'exception de l'Angleterre, des États-Unis et de l'Australie, où le taux de réponse fut de $3 \%, 6 \%$ et $11 \%$ respectivement, le pourcentage de questionnaires remplis fut relativement élevé, variant entre $20 \%$ pour le Canada et $64 \%$ pour la Finlande. Le taux de réponse des non-entrepreneurs fut deux fois plus élevé, près de $50 \%$. On

1 Les questionnaires en anglais ou en français sont disponibles sur demande. 
trouvera dans Scheinberg et MacMillan (1988) d'autres renseignements utiles sur l'enquête et la façon dont les échantillons nationaux furent construits.

Tab.1 Population étudiée

\begin{tabular}{|l|c|c|c|c|}
\hline \multicolumn{1}{|c|}{ Pays } & Acronyme & $\begin{array}{c}\text { Nombre } \\
\text { d'entrepreneurs }\end{array}$ & $\begin{array}{c}\text { Nombre de non- } \\
\text { ontrepreneurs }\end{array}$ & TOTAL \\
\hline Angleterre & ENG & 73 & - & 73 \\
Australie & AUS & 265 & 80 & 345 \\
Canada & CAN & 173 & 398 & 571 \\
Chine (R.P.C.) & CHI & 100 & 100 & 200 \\
Danemark & DEN & 77 & - & 77 \\
États-Unis & USA & 356 & 100 & 456 \\
Finlande & FIN & 116 & 134 & 250 \\
Itali** & ITA* & 163 & - & 163 \\
Kenya & KEN & 114 & - & 114 \\
Norvège & NOR & 148 & - & 148 \\
Québec** & QUE** & 331 & 527 & 858 \\
Portugal & POR & 122 & 122 & 244 \\
Porto Rico & PUE & 81 & 174 & 255 \\
Suède & SWE & 159 & 98 & 257 \\
\hline TOTAL & & 2278 & 1733 & 4011 \\
\hline
\end{tabular}

- Cet échantillon provient à $39 \%$ de régions pauvres de l'Italie. Voir Dubini (1988).

* Le Québec est classé séparément du Canada car les répondants sont tous de langue française alors que les répondants des autres provinces canadiennes sont tous de langue anglaise. L'échantillon canadien ( $n=173$ ) comprend 54 Québécois francophones $(31 \%)$, soit une proportion un peu supérieure à la population du Québec (26\%). Ces 54 Québécois ont été choisis au hasard et ont répondu au même questionnaire en anglais que les autres répondants canadiens.

\section{$5 \quad$ Résultats}

\subsection{Analyse des données}

Les données ${ }^{2}$ entrées sur ordinateur ont été soigneusement vérifiées au préalable et codifiées de la même façon quels que soient les pays. Elles ont ensuite été entrées dans les fichiers informatiques selon un format identique pour tous les individus.

2 Bien que cette étude ne couvre que 38 des 598 éléments d'information recueillis sur chaque entrepreneur, la banque de données qui en résulte est énorme et ne peut être traitée convenablement que par un puissant ordinateur, dans notre cas un IBM 3090-180, et à l'aide de logiciels éprouvés, dans notre cas le système SAS ainsi que le logiciel français ADDAD. Les analyses rapportées ici ont exigé plus d'un milliard d'opérations de calcul. 


\section{2 Énoncés de motivation}

Le tableau 2 fournit la liste complète des énoncés qui ont servi à mesurer les 38 motivations des entrepreneurs. Les chiffres entre parenthèses indiquent l'ordre dans lequel apparaissaient les énoncés dans tous les questionnaires.

Pour des raisons pratiques, nous avons classé les énoncés suivant une classification logique, qui n'estrien d'autre qu'un regroupement intuitif des variables selon les huit familles de motivations. Les acronymes utilisés facilitent le repérage des variables. Cette classification a priori pourra être comparée à la classification obtenue a posteriori par analyse factorielle (cf. tab.).

Tab.2 Classification logique des 38 énoncés de motivation

\begin{tabular}{|c|c|c|}
\hline \multicolumn{3}{|c|}{ Accomplissement personnel } \\
\hline ACC1 & (M28) & Atteindre une plus grande satisfaction personnelle. \\
\hline ACC2 & (M37) & $\begin{array}{l}\text { Prouver que je peux faire un succès d'une nouvelle } \\
\text { entreprise. }\end{array}$ \\
\hline ACC3 & (M7) & $\begin{array}{l}\text { Tirer meilleur profit de ma formation et de mes } \\
\text { talents. }\end{array}$ \\
\hline ACC4 & (M27) & $\begin{array}{l}\text { Relever le défi des problèmes et des opportunités } \\
\text { qu'occasionne la mise sur pied } \\
\text { d'une nouvelle entreprise. }\end{array}$ \\
\hline ACC5 & (M34) & Pour continuer d'apprendre. \\
\hline ACC6 & (M1) & $\begin{array}{l}\text { Prouver ma capacité de développer une nouvelle } \\
\text { idée. }\end{array}$ \\
\hline ACC7 & (M33) & $\begin{array}{l}\text { Etre innovateur et me tenir à la fine pointe des } \\
\text { technologies. }\end{array}$ \\
\hline ACC8 & (M31) & Suivre l'exemple de quelqu'un que j'admire. \\
\hline \multicolumn{3}{|c|}{ Désir d'indépendance } \\
\hline IND1 & (M6) & Etre mon propre patron; travailler pour moi-même. \\
\hline IND2 & (M38) & Jouir d'une pleine liberté vis-à-vis mon travail. \\
\hline IND3 & (M16) & Pour établir mon propre horaire de travail. \\
\hline IND4 & (M21) & Avoir l'opportunité de diriger, au lieu d'être dirigé. \\
\hline IND5 & (M15) & Pouvoir travailler avec des gens que je choisis. \\
\hline \multicolumn{3}{|c|}{ Opportunisme } \\
\hline OPP1 & (M26) & $\begin{array}{l}\text { A ce moment-là de ma vie, c'était la seule chose à } \\
\text { faire. }\end{array}$ \\
\hline OPP2 & (M3) & $\begin{array}{l}\text { Pour avoir plus de variété et d'aventure dans mon } \\
\text { travail. }\end{array}$ \\
\hline $\begin{array}{l}\text { OPP3 } \\
\text { OPP4 }\end{array}$ & $\begin{array}{l}(M 8) \\
(M 12)\end{array}$ & $\begin{array}{l}\text { Profiter d'une opportunité exceptionnelle. } \\
\text { Pour m'amuser. }\end{array}$ \\
\hline \multicolumn{3}{|c|}{ Besoin d'argent } \\
\hline ARG1 & (M23) & Pourvoir à ma subsistance et celle de ma famille. \\
\hline ARG2 & (M32) & Faire plus d'argent. \\
\hline ARG3 & (M2) & Besoin d'argent pour survivre. \\
\hline
\end{tabular}


ARG4 (M35) Obtenir des avantages sociaux.

Affiliation

AFF1 (M36) Avoir plus de flexibilité dans ma vie personnelle et familiale.

AFF2 (M25) Pouvoir travailler dans un milieu agréable pour moi et ma famille.

AFF3 (M22) Pouvoir travailler avec des gens que j'estime.

Reconnaissance sociale

REC1 (M14) Réaliser quelque chose et me faire reconnaitre en conséquence.

REC2 (M5) Atteindre un niveau plus élevé dans la société.

REC3 (M30) Faire partie d'un réseau d'entrepreneurs.

REC4 (M29) Hausser le statut et le prestige de ma famille.

REC5 (M13) Pour être respecté par mes amis.

REC6 (M11) Avoir plus d'influence dans mon milieu.

REC7 (M9) Désir de continuer la tradition familiale.

\section{Evasion}

EVA1 (M4) A cause de la frustration dans mon emploi précédent.

EVA2 (M24) Pour ne plus avoir à travailler pour un patron dérai-

sonnable.

EVA3 (M10) J'étais sans emploi et c'était la seule chose à faire.

EVA4 (M17) Pour échapper à des conditions de travail non sécuritaires.

\section{Altruisme communautaire}

ALT1 (M18) Assurer la sécurité et le bien-être de ma famille.

ALT2 (M20) Aider la communauté dans laquelle je vis.

ALT3 (M19) Pour contribuer au bien-être de mon groupe ethnique.

(les chiffres entre parenthèses indiquent l'ordre original des énoncés dans le questionnaire où ils sont numérotés de façon aléatoire)

\subsection{Analyse des fréquences}

Un aspect révélateur de l'importance relative des motivations, qui n'avait pas encore été étudié par les autres collègues du réseau SARIE, est l'analyse des fréquences des motivations selon les pays. Il s'agit tout simplement de calculer le nombre d'individus d'un pays donné qui ont répondu «très important» ou «important» à chacun des énoncés.

Cette analyse très simple révèle l'importance accordée à chacune des motivations, à la fois selon les individus et selon les pays. Elle est d'autant plus intéressante qu'elle exprime une réalité qui n'apparaît guère dans les analyṣes factorielles classiques : elle met en relief les ressemblances plutôt que les différences.

Avant le recodage, le pourcentage moyen des répondants ayant répondu «très important» ou «important» aux 38 énoncés de motivation oscille autour de 36 $\%$ alors que pour la Suède il est de $23 \%$ et pour Porto Rico de $58 \%$. Des différences 
plus importantes sont observées lorsqu'on examine des éléments particuliers. Il semble donc de prime abord que les répondants suédois étaient très réservés dans leurs réponses, la majorité d'entre eux hésitant à déclarer comme importante ou très importante telle ou telle motivation. Par contre, les répondants portoricains étaient très enthousiastes et choisissaient les extrêmes de l'échelle.

Ces différences et ressemblances deviennent plus évidentes lorsqu'on examine des groupes particuliers de variables. Par exemple, les répondants italiens et chinois se ressemblent passablement si on examine leurs fréquences de motivations. Mais si on considère l'autre extrême, à savoir le pourcentage de répondants qui n'attachent «aucune importance» à telle ou telle motivation, la ressemblance entre l'Italie et la Chine populaire devient encore plus évidente (cf. $t a b .3$ ).

\section{Tab.3 Similitudes des motivations entre l'Italie et la Chine}

\begin{tabular}{|llccc|}
\hline & Énoncés de motivation & Italie & Chine & Autres pays \\
\hline REC7 & $\begin{array}{l}\text { Pour continuer la tradition familiale } \\
\text { EVA4 }\end{array}$ & $\begin{array}{l}\text { Pour échapper à des conditions } \\
\text { de travail non sécuritaires }\end{array}$ & $62,6 \%$ & $81,7 \%$ \\
ALT3 & $\begin{array}{l}\text { Pour contribuer au bien-être de } \\
\text { mon groupe ethnique }\end{array}$ & $49,7 \%$ & $44,0 \%$ & $77,2 \%$ \\
\hline
\end{tabular}

(pourcentage de répondants ayant répondu «nullement importantw)

Au niveau des individus, le même phénomène existe : certains sont très prudents dans leur façon de répondre à un énoncé, d'autres tendent aux extrêmes. Comment donc tenir compte de ces biais personnels et collectifs?

\subsection{Recodage selon la méthode de l'équation personnelle}

Pour atténuer l'effet des façons de répondre, nous avons appliqué la méthode de Benzécri (1989), dite d'équation personnelle (Blais, Toulouse et Clément, 1990). Cette méthode permet d'enlever les «irrégularités"» dans les réponses individuelles et assure l'homogénéité des données qui s'avère si nécessaire dans les comparaisons internationales de résultats d'enquête. La corrélation entre les réponses originales et recodées est de 0,78 .

\subsection{Analyse des fréquences des données de motivation recodées}

Suite au recodage et à la reclassification des données par la méthode de l'équation personnelle, nous avons recalculé les fréquences des motivations selon les 


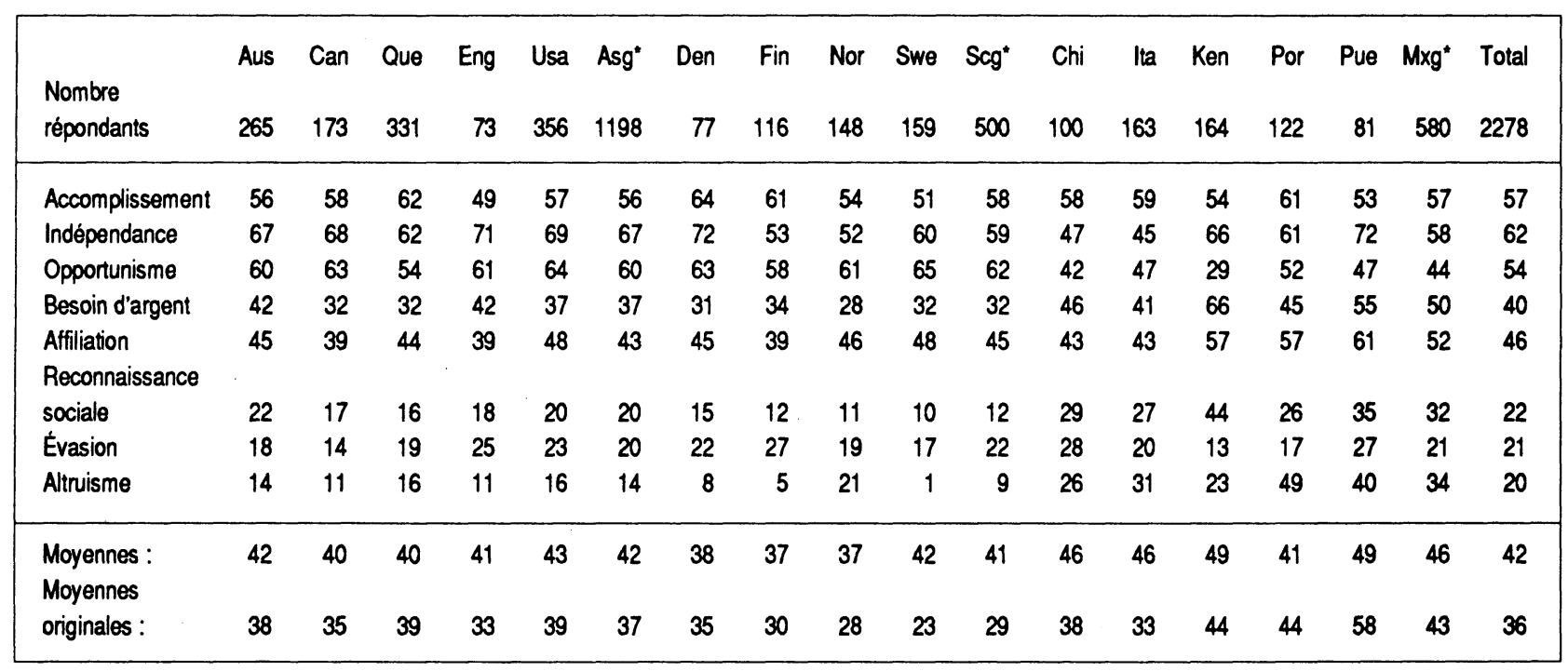

(il s'agit du pourcentage des répondants qui ont répondu «très important" ou «important» aux énoncés du questionnaire, ces données ayant été recodées et reclassées par la méthode de l'équation personnelle)

ASG : Moyenne pour les pays anglo-saxons, SCG : pays scandinaves, MXG : pays du groupe mixte.

*. Les variables constituant chaque famille de motivations sont énumérées dans le tableau 2. 
pays, i.e. le pourcentage des répondants ayant répondu «très important» ou «important à chacun des énoncés. Le tableau 4, p. 281, donne les fréquences moyennes des motivations regroupées en familles (cf. tab.2) pour chaque groupe national de répondants. L'emplacement des colonnes est justifié par les résultats de l'analyse hiérarchique (cf. section 4.๑). L'ordre des rangées est le même que celui qui apparait dans la «classification logique» des variables (tab.2).

Les résultats du recodage des fréquences se résument comme suit :

- en dépit de différences culturelles fondamentales, les répondants québécois (de langue française) expriment exactement les mêmes motivations que les répondants canadiens (de langue anglaise), ce qui donne déjà à penser que l'entrepreneuriat transcende les cultures et s'insère plutôt dans l'environnement économique et la reconnaissance sociale (cf. tab. 5);

- d'autre part, les grandes différences entre les répondants suédois et portoricains s'atténuent : la différence passe de $34,8 \%$ à $12,5 \%$ pour les cotes 1 et 2 , et de $31,8 \%$ à $19,0 \%$ pour les cotes 4 et 5 ;

- les motivations de développement personnel, d'indépendance et d'opportunité reçoivent des cotes élevées dans tous les pays mais à des degrés divers selon les régions;

- les motivations d'argent et d'affiliation sont moyennement importantes, excepté dans les pays en voie de développement où elles prévalent;

- la reconnaissance sociale et l'altruisme communautaire sont peu cités comme motivations dans la plupart des pays sauf les pays en voie de développement et l'Italie, alors que l'évasion est faible partout.

Tab. 5 Effet du recodage, par équation personnelle, sur les fréquences moyennes de l'ensemble des motivations des entrepreneurs canadiens, québécols, suédois et portoricains

\begin{tabular}{|c|c|c|c|c|c|c|c|c|c|c|}
\hline Pays & $\begin{array}{l}\quad 1 \\
\text { Très im } \\
\text { Avant }\end{array}$ & $\begin{array}{l}\text { 1) } \\
\text { nportant } \\
\text { Après }\end{array}$ & $\begin{array}{r}\text { (2) } \\
\text { t Impo } \\
\text { Avant }\end{array}$ & $\begin{array}{l}\text { 2) } \\
\text { Aprant } \\
\text { Après }\end{array}$ & Assez & $\begin{array}{l}\text { (3) } \\
\text { importar } \\
\text { Après }\end{array}$ & $\begin{array}{l}\text { ntPeu in } \\
\text { Avant }\end{array}$ & $\begin{array}{l}\text { (4) } \\
\text { importar } \\
\text { Après }\end{array}$ & $\begin{array}{l}\text { Avant } \\
\text { Avas }\end{array}$ & $\begin{array}{l}\text { (5) } \\
\text { mportant } \\
\text { Après }\end{array}$ \\
\hline $\begin{array}{l}\text { Canada } \\
\text { (N=173) } \\
\text { Québec } \\
\text { (N=331) } \\
\text { Suède } \\
\text { (N=159) } \\
\text { Porto Rico } \\
\text { (N=81) }\end{array}$ & $\begin{array}{r}14,6 \\
16,6 \\
11,6 \\
35,0\end{array}$ & $\begin{array}{r}18,6 \\
19,9 \\
17,8 \\
35,1\end{array}$ & $\begin{array}{r}19,8 \\
18,8 \\
12,4 \\
23,8\end{array}$ & $\begin{array}{r}21,0 \\
19,8 \\
18,8 \\
14,0\end{array}$ & $\begin{array}{l}24,4 \\
23,6 \\
19,3 \\
16,3\end{array}$ & $\begin{array}{r}20,2 \\
19,6 \\
11,0 \\
17,5\end{array}$ & $\begin{array}{c}14,4 \\
11,9 \\
11,6 \\
10,6\end{array}$ & $\begin{array}{r}13,0 \\
11,4 \\
6,1 \\
13,7\end{array}$ & $\begin{array}{r}26,8 \\
29,1 \\
45,1 \\
14,3\end{array}$ & $\begin{array}{r}27,2 \\
29,3 \\
46,3 \\
19,7\end{array}$ \\
\hline
\end{tabular}

(les chiffres indiqués dans les colonnes sont des pourcentages du total des réponses pour l'ensemble des 38 motivations pour chaque pays) 


\subsection{Analyse hiérarchique}

Afin de dégager la structure sous-jacente aux motivations dans les divers pays, nous nous sommes servi du logiciel français ADDAD pour l'analyse hiérarchique. Cette méthode, dite des «voisins réciproques», fournit un degré d'agrégation de plus en plus élevé des variables.

La classification hiérarchique ascendante (CHA) des 38 motivations dans chaque pays donne les résultats illustrés à la figure 1, où l'abcisse indique le niveau croissant d'agrégation. Plus le niveau est bas, plus le «profil de motivation» des pays qui se situent à ce niveau est semblable. Par exemple, l'Australie, le Canada et les États-Unis affichent le profil le plus semblable, et la Chine et le Kenya le plus différent. Cette classification a été faite sur l'ensemble des données de fréquences alors que le tableau 3 ne donne que les moyennes correspondant aux «familles» de motivations ( $c f . t a b .2)$.

Fig.1 Analyse hiérarchique des profils nationaux de motivations entrepreneuriales

\section{NIVEAU CROISSANT D'AGRÉGATION}

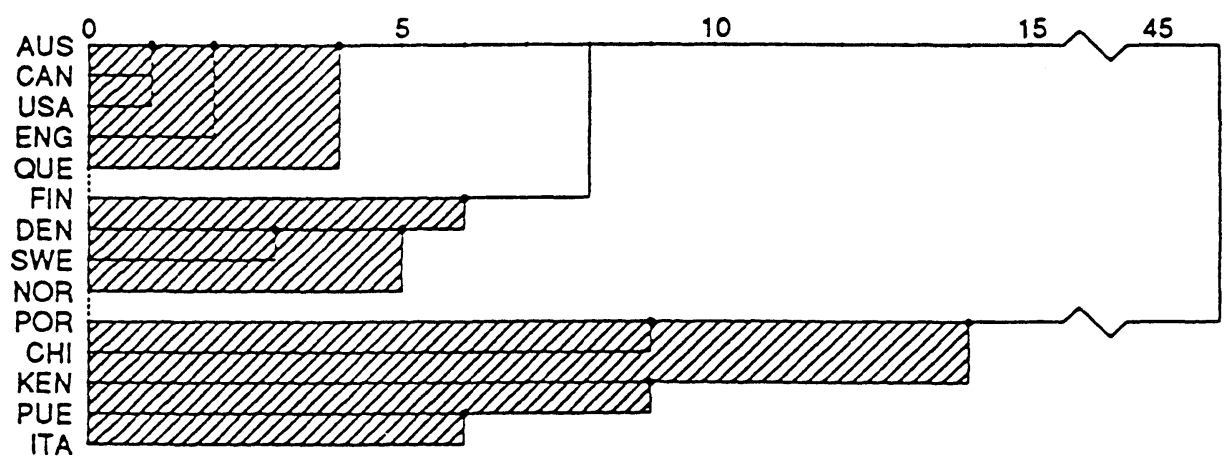

L'analyse hiérarchique des données permet d'identifier clairement trois groupes régionaux : le bloc anglo-saxon, le bloc scandinave et le bloc mixte. Cette analyse mène aux conclusions suivantes :

1. Les entrepreneurs des pays anglo-saxons ont essentiellement les mêmes motivations. Toutefois, les entrepreneurs francophones du Québec se classent entre les Anglo-saxons et les Scandinaves. Notons que la province de Québec affiche des tendances vers la social-démocratie et qu'avec la faillite de «l'accord du lac Meech» en juin 1990 cette province montre plus que jamais un désir de souveraineté politique. 
2. Les Scandinaves forment un autre groupement naturel, i.e. les entrepreneurs de ces quatre pays invoquent les mêmes motivations.

3. Le troisième groupement naturel est celui des entrepreneurs des pays en voie de développement (ou moins industrialisés). Les entrepreneurs italiens appartiennent à ce groupe, car plus du tiers de ces répondants oeuvrent dans les parties pauvres de l'Italie.

\subsection{Analyse factorielle}

L'univers des motivations des 2278 entrepreneurs fut soumis à l'analyse factorielle en composantes principales (ACP) afin de trouver les groupements de variables fortement corrélées entre elles, ainsi que la distribution de ces groupements selon les pays. Nous cherchions en quelque sorte à édifier, à l'aide des données recodées, un modèle mondial des motivations entrepreneuriales.

Après divers essais, incluant les méthodes Varimax, Quartimax, Promax et autres, avant et après rotation oblique et orthogonale, nous avons finalement opté pour la méthode Varimax après rotation orthogonale des axes. Puis, nous avons essayé diverses techniques pour obtenir le meilleur nombre de facteurs, par exemple en éliminant successivement les facteurs n'ayant pas plus d'une variable avec un poids («factor loading») supérieur à 0,40 . La technique intégrative que nous avons utilisée consiste à se servir des 38 variables au lieu d'en éliminer 17, comme l'ont fait Scheinberg et MacMillan (1988), de façon à mieux représenter la totalité du phénomène à élucider. Il s'agissait ensuite de construire l'histogramme des valeurs propres à la matrice, i.e. d'appliquer le «scree test», afin de déterminer le nombre de facteurs à retenir.

La meilleure solution s'est avérée être 7 facteurs. Pour fins de clarté de présentation, nous n'avons indiqué que les pondérations supérieuresà 0,40 (cf. tab.Ø). Nous avons par la suite éliminé trois variables ne s'identifiant à aucun des facteurs, à savoir :

OPP3 : Profiter d'une opportunité exceptionnelle.

OPP4: Pour m'amuser.

REC3 : Faire partie d'un réseau d'entrepreneurs.

Les sept facteurs retenus sont, en ordre décroissant d'importance : la reconnaissance sociale (F1), l'accomplissement personnel (F2), l'argent (F3), le besoin de pouvoir, d'autonomie et la liberté de pouvoir choisir (F4), l'altruisme communautaire (F5), l'évasion (F6) et l'opportunisme (F7) [cf. tab.6]. Ces facteurs permettent d'expliquer $50,8 \%$ de la variance totale ce qui, pour 2278 individus de 14 pays, se compare favorablement au $64 \%$ obtenu par Scheinberg et MacMillan (1988) pour 1400 entrepreneurs de onze pays seulement, et pour seulement 21 variables comparativement à 35 dans notre cas (compte tenu de la suppression des trois variables susmentionnées). 
Tab.6 Analyse factorielle des motivations de 2278 entrepreneurs dans 14 pays, fondée sur des données recodées et reclassées par la méthode de l'équation personnelle

\begin{tabular}{|c|c|c|c|c|c|c|c|}
\hline Variables de motivation & F1 & F2 & F3 & F4 & F5 & F6 & F7 \\
\hline \multicolumn{8}{|l|}{ Besoin de reconnaissance sociale } \\
\hline REC5 Pour être respecté par mes amis & 0,74 & - & - & - & - & - & - \\
\hline REC6 Avoir plus d'influence dans mon milieu & 0,62 & - & - & - & - & - & - \\
\hline ACC8 Suivre l'exemple de quelqu'un que j'admire & 0,58 & - & - & - & - & - & - \\
\hline \multicolumn{8}{|l|}{ REC4 Hausser le statut et le prestige de } \\
\hline ma famille & 0,56 & - & - & - & - & - & - \\
\hline \multicolumn{8}{|l|}{ REC2 Atteindre un niveau plus élevé dans } \\
\hline la société & 0,52 & - & - & - & - & - & - \\
\hline \multicolumn{8}{|l|}{ REC1 Atteindre une plus grande satisfaction } \\
\hline personnelle & 0,49 & - & - & - & - & - & - \\
\hline \multicolumn{8}{|l|}{ Besoin d'accomplissement personnel } \\
\hline \multicolumn{8}{|l|}{ ACC7 Etre innovateur et à la fine pointe des } \\
\hline technologies & - & 0,70 & - & - & - & - & - \\
\hline ACC5 Pour continuer d'apprendre & - & 0,70 & - & - & - & - & - \\
\hline \multicolumn{8}{|l|}{ ACC6 Prouver ma capacité de développer une } \\
\hline nouvelle idée & - & 0,56 & - & - & - & - & - \\
\hline \multicolumn{8}{|l|}{ ACC2 Prouver que je peux réussir dans } \\
\hline une nouvelle entreprise & - & 0,48 & - & - & - & - & - \\
\hline \multicolumn{8}{|l|}{ ACC3 Tirer meilleur profit de ma formation } \\
\hline et de mes talents & - & 0,40 & . & - & - & - & - \\
\hline \multicolumn{8}{|l|}{ Besoin d'argent } \\
\hline ARG2 Faire plus d'argent & & - & $-0,69$ & . & - & - & \\
\hline \multicolumn{8}{|l|}{ ARG1 Pourvoir à ma subsistance ef celle } \\
\hline de ma famille & - & - & $-0,65$ & - & - & - & \\
\hline ARG3 Besoin d'argent pour survivre & - & - & $-0,61$ & - & - & - & \\
\hline \multicolumn{8}{|l|}{ Besoin de pouvoir } \\
\hline \multicolumn{8}{|l|}{ IND1 Etre mon propre patron : travailler } \\
\hline pour moi-même & - & - & - & 0,57 & - & - & - \\
\hline \multicolumn{8}{|l|}{ OPP2 Avoir plus de variété et d'aventure } \\
\hline dans mon travail & - & . & - & 0,52 & - & - & . \\
\hline \multicolumn{8}{|l|}{ IND4 Avoir l'opportunité de diriger, au lieu } \\
\hline d'être dirigé & - & - & - & 0,52 & - & - & - \\
\hline \multicolumn{8}{|l|}{ ACC1 Atteindre une plus grande satisfaction } \\
\hline personnelle & - & - & - & 0,47 & - & - & - \\
\hline \multicolumn{8}{|l|}{ EVA3 J'étais sans emploi et c'était la seule } \\
\hline chose à faire & - & - & - & $-0,43$ & - & - & - \\
\hline \multicolumn{8}{|l|}{ Besoin d'autonomie } \\
\hline \multicolumn{8}{|l|}{ AFF1 Avoir plus de latitude dans ma vie } \\
\hline personnelle et familiale & - & - & - & 0,72 & - & - & - \\
\hline IND3 Pour établir mon propre horaire de travail & - & - & - & 0,66 & - & - & $\therefore$ \\
\hline IND2 Jouir d'une pleine liberté vis-à-vis mon travail & - & - & . & 0,61 & - & - & . \\
\hline ARG4 Obtenir des bénéfices marginaux & - & - & - & 0,41 & - & - & - \\
\hline
\end{tabular}




$\begin{aligned} & \text { Avoir la liberté de choisir } \\ & \text { AFF3 Pouvoir travailler avec des gens que j'estime } \\ & \text { IND5 Pouvoir travailler avec des gens que je choisis }\end{aligned}$ -
$\begin{aligned} & \text { Altruisme communautaire } \\ & \text { ALT3 Pour contribuer au bien-être de }\end{aligned}$
$\quad$ mon groupe ethnique

(les points indiquent des pondérations inférieures à 0,4 )

* A eux-seuls, ces trois sous-facteurs (inclus sous F4) permettent d'expliquer $12,37 \%$ de la variance totale.

* Valeur Eigen moyenne des trois sous-facteurs (indépendance, autonomie et avoir la liberté de choisir).

\subsection{Discussion des groupes factoriels de motivation}

L'image des motivations entrepreneuriales qui ressort du tableau 6 est très instructive, car sept facteurs sont clairement identifiés. Ces facteurs confirment ce que de nombreux chercheurs ont affirmé empiriquement ou intuitivement.

Cette image globale se rapproche de celle élaborée par le sociologue suédois Friberg (1976). Selon ce chercheur, les entrepreneurs partagent l'une ou plusieurs des quatre motivations suivantes :

- Nécessité de reconnaissance sociale et du désir d'identification et d'implication.

- Nécessité d'avoir de l'argent.

- Nécessité d'accommodation personnelle et désir de défis technologiques ou commerciaux.

- Nécessité d'échappement causé par la coercition. 
Il manque cependant à cette liste le besoin très important d'indépendance, d'autonomie, reconnu par presque tous les auteurs. Il faudrait aussi y ajouter l'esprit communautaire et le collectivisme qui poussent certains individus à entrer en affaires. Quant à notre septième et plus faible facteur — l'opportunisme - il n'est pas très distinctif et ne correspond pas à une théorie particulière.

Les groupes factoriels de motivation obtenus appellent les commentaires suivants :

\section{FACTEUR 1-DÉSIR DE RECONNAISSANCE SOCIALE}

Ce facteur regroupe des variables qui ont trait au statut, au prestige que l'entrepreneur reçoit de la part des autres membres de la société. Il traduit également le désir d'être respecté et admiré par les amis et les proches, parce que l'on est entrepreneur. En fait, c'est un peu le désir d'être remarqué par les autres. Ce facteur traduit un mélange de besoins d'affiliation et de pouvoir tels que formulés par McClelland (1961). Il épouse aussi la théorie de Maslow (1954) sur le besoin d'estime qu'éprouvent les gens.

\section{FACTEUR 2 - DÉSIR D'ACCOMPLISSEMENT PERSONNEL}

Cette motivation exprime la volonté de relever des défis, de prouver qu'on est capable de faire un succès d'une entreprise. Elle traduit aussi le désir d'être innovateur, de développer de nouvelles idées, de créer de nouveaux produits. Ce facteur correspond au besoin d'accomplissement dans la théorie de McClelland (1961) et à celui de réalisation dans la théorie de Maslow (1954).

\section{FACTEUR 3 - NÉCESSITÉ D'AVOIR DE L'ARGENT}

Le désir de faire de l'argent est une motivation significative chez certains entrepreneurs, surtout ceux et celles qui ont perdu leur emploi ou qui cherchent à mieux subvenir à leurs besoins et à ceux de leur famille. L'argent est également une préoccupation importante pour les entrepreneurs jeunes, les immigrants et les opportunistes. Cependant, tel que souligné par Gasse (1982) et Toulouse (1979), très souvent la motivation pécuniaire ne compte pas beaucoup dans la décision de fonder une entreprise. Dans le même sens, Blais et Toulouse (1989) ont trouvé que les entrepreneurs québécois $(\mathrm{N}=331)$ sont peu motivés par le motif intrinsèque de l'argent alors que c'est le contraire pour les non-entrepreneurs $(\mathrm{N}=526)$.

\section{FACTEUR 4 - BESOIN DE POUVOIR, D'AUTONOMIE ET DESSIR DE LI- BERTÉ}

Ce facteur souligne le besoin d'autonomie des individus qui désirent le pouvoir, qui veulent diriger, être leur propre patron, choisir ce qu'il faut faire, maîtriser leur temps, régler eux-mêmes leur travail, choisir ceux qu'ils préferent, en un mot mieux contrôler leur destinée, être aux commandes et influencer ce qui leur arrive. On retrouve souvent cette motivation chez les entrepreneurs artisans : ils veulent agir selon leurs désirs, en suivant leurs aspirations et leurs goûts. Cette notion très forte rejoint le concept d'individualisme de Hofstede (1980) selon lequel le contrôle de sa 
destinée et la liberté de choix sont des objectifs personnels importants. Elle coïncide également avec la théorie de contrôle interne de la situation («Internal focus of control») développée par plusieurs auteurs, dont Rotter (1964). La pondération négative que reçoit la variable EVA3 sous le facteur F4 montre le déterminisme de l'entrepreneur. Pour lui, fonder une entreprise est l'antithèse de ne pas avoir d'autre chose à faire.

\section{FACTEUR 5 - ALTRUISME COMMUNAUTAIRE}

Ce facteur regroupe des éléments qui indiquent une motivation à travailler au bienêtre de la collectivité ou de son groupe ethnique pour aider ses proches et assurer la sécurité et le bien de sa famille. On retrouve cette motivation chez les entrepreneurs qui oeuvrent dans le domaine social et, surtout, dans les cultures qui valorisent le groupe plutôt que l'individu. Ce facteur rejoint les postulats de Hofstede (1980) sur le collectivisme.

\section{FACTEUR 6 - BESOIN D'ÉVASION}

Plusieurs auteurs ont souligné l'importance du phénomène de «déplacement» dans la création de nouvelles entreprises. Pour toutes sortes de raisons, certains individus à un moment donné se sentent mal dans leur peau et désirent faire autre chose : certains sont dégoûtés de leur emploi, d'autres ont tout simplement perdu leur emploi, d'autres encore recherchent de meilleures conditions, etc. Collins et al. (1970) et Cooper (1971) ont montré que les expériences de travail négatives comptent pour beaucoup dans la décision de partir en affaires. Friberg (1976) a également montré que souvent des facteurs négatifs ou des forces coercitives entrent en jeu dans la décision de fonder une entreprise. Ces concepts rejoignent d'ailleurs les idées de Shapero (1975) sur l'individu «non confortable» qui devient, un peu malgré lui, entrepreneur.

\section{FACTEUR 7 -OPPORTUNISME}

Les trois éléments représentant ce facteur (cf. tab. 6, $2 \%$ de la variance totale) se rapportent à des opportunités qui se sont présentées : les conditions étaient appropriées pour partir en affaires, une bonne occasion s'est présentée, on est parti en affaires pour s'offrir un milieu agréable ainsi qu'à sa famille.

\subsection{Cotes factorielles moyennes pour les 14 pays}

Une des facettes les plus intéressantes de cette recherche est le «positionnement» de chacun des 14 pays par rapport aux groupes factoriels obtenus (cf. tab. 6). Autrement dit, dans quelle mesure les entrepreneurs d'un pays partagentils les mêmes motivations que les entrepreneurs d'un autre pays ? Et si des agglomérations de groupes d'entrepreneurs se produisent autour de certaines motivations, quelle en est la cause ? Que peut-on en conclure? 
Le tableau 7 montre les cotes factorielles moyennes («mean factor scores») des divers pays étudiés. Compte tenu de l'ordre inversé dans l'échelle Likert utilisée, où $1=$ le plus important et $5=$ pas important, plus les cotes sont négatives, plus ce facteur est important pour les entrepreneurs du pays alors que c'est le contraire lorsque les cotes sont positives.

Tab. 7 Cotes factorielles moyennes pour les 14 pays

\begin{tabular}{|c|c|c|c|c|c|c|c|c|}
\hline Pays & $N$ & $\begin{array}{l}\text { Reconnais- } \\
\text { sociale }\end{array}$ & $\begin{array}{l}\text { Accom- } \\
\text { plissement } \\
\text { personnel }\end{array}$ & Argent & $\begin{array}{l}\text { Pouvoir et } \\
\text { autonomie }\end{array}$ & $\begin{array}{c}\text { Altruisme } \\
\text { communau- } \\
\text { taire }\end{array}$ & Évasion & $\begin{array}{c}\text { Opportu } \\
\text { nisme }\end{array}$ \\
\hline Australie & 265 & $-0,05$ & $-0,03$ & $-0,09$ & $-0,17$ & $-0,41$ & $+0,27$ & $+0,27$ \\
\hline Canada & 173 & $-0,01$ & $-0,08$ & $-0,16$ & $-0,17$ & $+0,15$ & $+0,24$ & $+0,18$ \\
\hline $\begin{array}{l}\text { États-Unis } \\
+0,08\end{array}$ & 356 & & 0,00 & $-0,17$ & $-0,04$ & $-0,22$ & $-0,13$ & $-0,13$ \\
\hline Angleterre & 73 & $+0,20$ & $+0,43$ & $-.0,06$ & $-0,20$ & $-0,45$ & $-0,23$ & $+0,22$ \\
\hline Québec & 331 & $+0,06$ & $-0,16$ & $+0,04$ & $-0,02$ & $-0,02$ & $-0,14$ & $-0,23$ \\
\hline Finlande & 116 & $+0,42$ & $-0,33$ & $-0,08$ & $+0,40$ & $+0,40$ & $-0,60$ & $-0,21$ \\
\hline Danemark & 77 & $+0,20$ & $-0,02$ & $+0,59$ & $-0,23$ & $+0,36$ & $+0,03$ & $-0,48$ \\
\hline Suède & 159 & $+0,83$ & $+0,52$ & $+0,38$ & $-0,09$ & $+0,45$ & $+0,39$ & $-0,40$ \\
\hline Norvège & 148 & $+0,44$ & $+0,03$ & $+0,44$ & $+0,21$ & $-0,26$ & $+0,07$ & $-0,07$ \\
\hline Portugal & 122 & $-0,08$ & $-0,22$ & $-0,09$ & $-0,03$ & $-1,06$ & $+0,16$ & $+0,64$ \\
\hline Chine & 100 & $-1,07$ & $-0,09$ & $+0,16$ & $+0,42$ & $-0,24$ & $-0,47$ & $+0,35$ \\
\hline Kenya & 164 & $-1,01$ & $+0,02$ & $-1,06$ & $-0,07$ & $-0,67$ & $+0,33$ & $+0,52$ \\
\hline Porto Rico & 81 & $-0,47$ & $-0,14$ & $-0,57$ & $-0,20$ & $-1,00$ & $-0,14$ & $-0,12$ \\
\hline Italie & 163 & $-0,17$ & $-0,08$ & $-0,40$ & $+0,53$ & $-0,56$ & $+0,10$ & $-0,46$ \\
\hline
\end{tabular}

(obtenues par analyse factorielle Varimax après rotation à l'orthogonale; données recodées et reclassées par la méthode d'équation personnelle)

On voit, à l'examen du tableau 7, le même regroupement de pays que celui révélé par la classification hiérarchique ascendante (CHA) des fréquences de motivation, c'est-à-dire que les cotes factorielles obtenues pour chaque pays conduisent au même regroupement bien qu'il s'agisse de deux méthodes fort différentes. Par exemple :

- le bloc anglo-saxon se distingue par un désir de pouvoir et d'autonomie très élevé (à l'exception du Québec) et le peu d'importance accordé à l'opportunisme. L'altruisme communautaire y est significatif, quoiqu'à un degré beaucoup moindre que dans les pays en voie de développement, au Portugal et en Italie. Le désir d'argent constitue un trait faiblement distinctif des entrepreneurs anglo-saxons, à l'exception du Québec qui penche plutôt du côté scandinave comme c'est le cas également pour l'opportunisme. Les entrepreneurs anglais semblent moins motivés par le désir d'accomplissement personnel et par le besoin de statut social que leurs homologues anglo-saxons.

- le bloc scandinave se distingue très nettement par le manque quasi total 
d'intérêt pour la reconnaissance sociale (sans doute parce que, dans ces quatre pays de social-démocratie, un niveauélevé de statut social est déjà atteint), par le manque généralisé d'intérêt pour l'argent comme raison majeure d'entrer en affaires (ces pays ont un niveau de vie élevê), par l'absence généralisée de besoin d'évasion (à l'exception de la Finlande) et, surtout, par l'attrait singulier des opportunités.

- le bloc mixte est tout aussi distinct : un désir très fort de reconnaissance sociale (surtout en Chine et au Kenya), un altruisme communautaire exceptionnellementélevé (particulièrement au Portugal et à Porto Rico) et, àl'instar des entrepreneurs anglo-saxons, le désir de faire de l'argent, de devenir riche.

Pour faciliter la perception de ces relations, nous avons tracé dans les figures 2 à 9 , p. 292 et 293, la position des divers pays relativement aux divers facteurs. On voit que dans la plupart des cas, trois agglomérations régionales apparaissent, soit les mêmes blocs anglo-saxon, scandinave et mixte qu'a révélés l'analyse CHA et qui sont caractérisés par les contributions relatives des facteurs figurant au tableau 7. Puisque le nombre des répondants anglo-saxons est dominant, i.e. 1198 sur 2278 $(52,6 \% \mathrm{du}$ total $)$, on comprendra que l'agglomération anglo-saxonne se trouve près du centre de gravité de l'univers étudié. Alors que les frontières des nuages de points sont approximatives et données seulement à titre d'illustration, la position des points par contre est exacte et correspond aux données énumérées dans le tableau 7.

Ces graphiques servent surtout à montrer de façon simple la distribution régionale des groupes factoriels de motivation. Par exemple, les motivations les plus fortes se trouvent dans le quadrant Nord-Est et les plus faibles dans le quadrant SudOuest. Ainsi, la figure 9 montre que l'argent est une forte motivation au Kenya, à Porto Rico, en Italie et un peu au Portugal, mais sans importance en République populaire de Chine, alors que dans tous ces pays l'altruisme communautaire est fort répondu, ce qui est loin d'être le cas en Suède, Finlande et Danemark.

En ce qui concerne le bloc scandinave, on voit par ces graphiques que les entrepreneurs suédois sont généralement les moins motivés quelles que soient les motivations considérées (ils se retrouvent presque toujours au bas dans le coin SudOuest des graphiques) alors que les entrepreneurs norvégiens sont plus intéressés à aider leur communauté et les entrepreneurs finlandais plus motivés par l'argent.

\subsection{Analyse des correspondances multiples}

Après avoir fait la classification hiérarchique ascendante (CHA) des données de fréquence (cf. section 5.6), ainsi que l'analyse factorielle en composantes principales (ACP) sur l'ensemble des 38 motivations (cotées de 1 à 5) des 2278 répondants ( $c f$. section 5.7), nous avons fait appel à l'analyse des correspondances multiples (ACM) pour faire ressortir les ressemblances et différences dans cette 
énorme masse de données. Après avoir vainement tenté de traiter 432820 points (i.e. $2278 \times 38 \times 5$ ), en disjonctif complet, nous avons réduit l'information en construisant un tableau de contingences pour les pays seulement. Nous avons adopté la même méthode de calcul des fréquences que dans la $\mathrm{CHA}$ et avons réduit le tableau à 532 points (i.e. $38 \times 14$ ) après avoir calculé les pourcentages de répondants ayant indiqué «très important» ou «important» sous les diverses rubriques de motivation.

Après avoir tracé l'histogramme des valeurs propres (un «scree test») de la matrice ACM, nous n'avons retenu que les trois premiers des 12 axes obtenus car à eux seuls ils expliquent $70,17 \%$ de la variance totale. Ces trois axes sont caractérisés par des variables différentes de celles qui composent en grande partie les axes factoriels ACP. Dans le tableau 8 , nous ne montrons que les variables apportant une contribution relative égale ou supérieure à $2,6 \%$ (i.e. $100 \%+38$ variables).

La projection des 14 pays sur les axes factoriels ACM montre les trois mêmes grandes agglomérations régionales qu'avaient révélées l'analyse ACP et l'analyse CHA. Cette découverte est d'autant plus intéressante qu'il s'agit là de trois méthodes fort différentes, ce qui illustre bien la robustesse du phénomène. La position relative des pays sur les axes $\mathrm{ACM} \mathrm{n}^{\circ} 1$ (en abscisse) et $\mathrm{n}^{\circ} 2$ (en ordonnée) se trouve illustrée dans la figure 10, p. 294. Il est à noter qu'on obtient la même distribution en trois entités régionales en projetant les pays sur les axes 1 et 3 . Par contre, la projection sur les axes 2 et 3 ne donne aucune image compréhensible.

Tab. 8 Définition des axes factoriels de correspondances multiples

\begin{tabular}{|c|c|c|c|c|c|c|c|c|}
\hline \multicolumn{3}{|c|}{ AXE $1(45,41 \%)$} & \multicolumn{3}{|c|}{ AXE $2(15,66 \%)$} & \multicolumn{3}{|c|}{ AXE $3(9,10 \%)$} \\
\hline $\begin{array}{l}\text { Moti- } \\
\text { vation }\end{array}$ & $\begin{array}{l}\text { Coordon- } \\
\text { née }\end{array}$ & $\begin{array}{l}\text { Contri- } \\
\text { bution }\end{array}$ & $\begin{array}{l}\text { Moti- } \\
\text { vation }\end{array}$ & $\begin{array}{l}\text { Coordon- } \\
\text { née }\end{array}$ & $\begin{array}{l}\text { Contri- } \\
\text { bution }\end{array}$ & $\begin{array}{l}\text { Moti- } \\
\text { vation }\end{array}$ & $\begin{array}{l}\text { Coordon- } \\
\text { née }\end{array}$ & $\begin{array}{l}\text { Contri- } \\
\text { bution }\end{array}$ \\
\hline EVA1 & +358 & 4,9 & REC5 & +486 & $13,3 \%$ & OPP4 & +317 & $26,4 \%$ \\
\hline OPP3 & +258 & 4,8 & ALT3 & +332 & 4,6 & REC3 & +289 & 22,1 \\
\hline OPP1 & +238 & 4,6 & OPP4 & +269 & 11,0 & ARG3 & +122 & 3,2 \\
\hline OPP2 & +220 & 3,6 & ALT2 & +246 & 4,0 & OPP2 & -109 & 4,5 \\
\hline ALT1 & -313 & 3,8 & EVA2 & +155 & 2,9 & ARG2 & -120 & 4,2 \\
\hline ARG3 & -323 & 5,2 & ALT1 & +153 & 2,6 & ALT1 & -142 & 3,9 \\
\hline ALT2 & -423 & 4,5 & ACC5 & +151 & 5,4 & EVA2 & -146 & 4,4 \\
\hline REC3 & -441 & 5,2 & $\mathrm{ACC} 2$ & +137 & 4,5 & OPP3 & -173 & 10,8 \\
\hline EVA4 & -506 & 3,5 & IND1 & -121 & 4,1 & ACC8 & -247 & 5,1 \\
\hline REC5 & -539 & 5,6 & OPP2 & -161 & 5,6 & REC5 & -293 & 8,3 \\
\hline REC6 & -598 & 7,2 & ARG2 & -171 & 5,4 & & & \\
\hline REC4 & -612 & 11,7 & IND4 & -194 & 7,5 & & & \\
\hline ACC8 & -686 & 7,9 & REC3 & -439 & 14,9 & & & \\
\hline ALT3 & -819 & 9,6 & & & & & & \\
\hline \multicolumn{3}{|c|}{14 variables $=82,1 \%$} & \multicolumn{3}{|c|}{13 variables $=85,8 \%$} & \multicolumn{3}{|c|}{10 variables $=81,9 \%$} \\
\hline
\end{tabular}


Fig. 2 à 9 Position des pays sur les divers facteurs de motivation
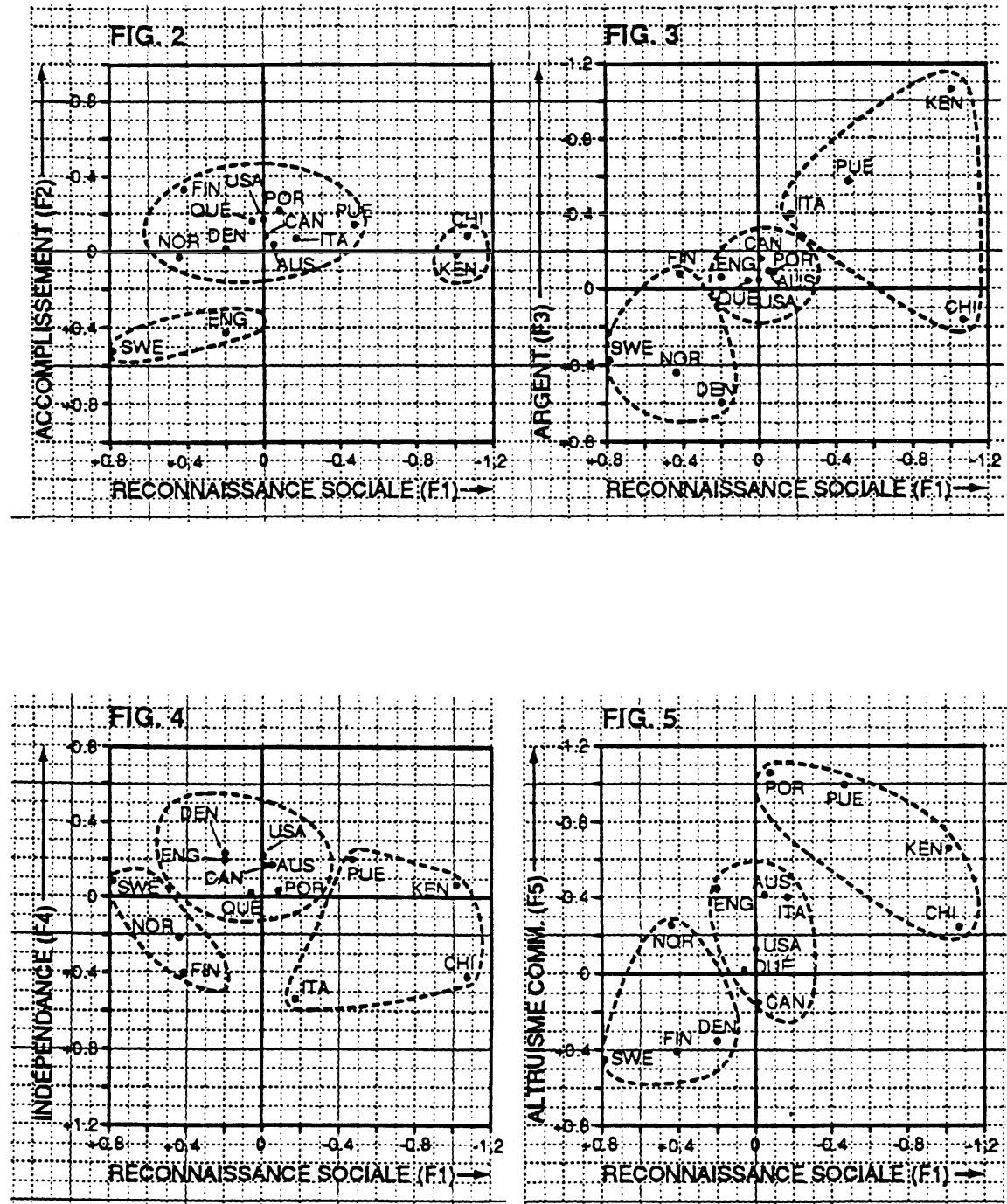

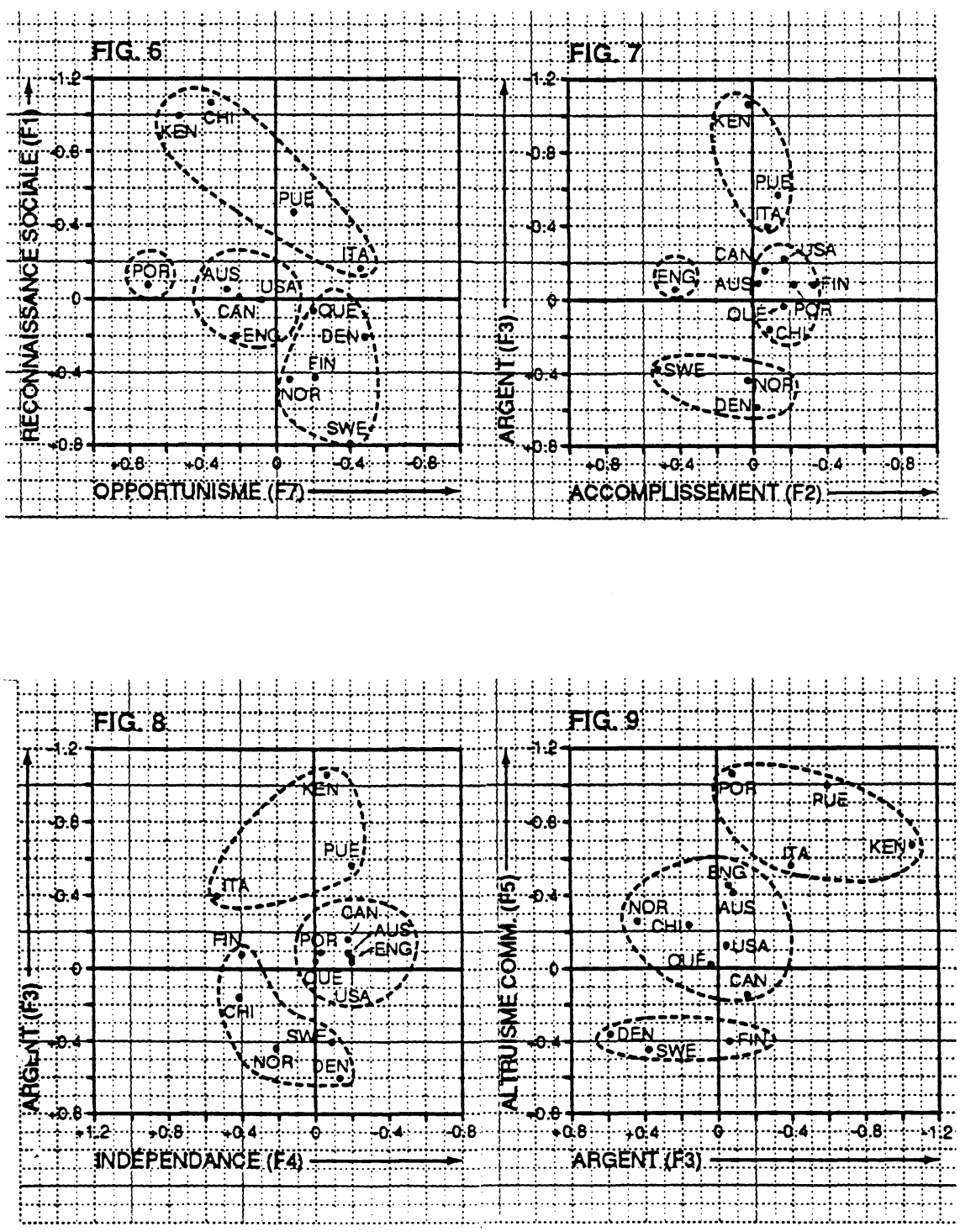
Fig. 10 Position des pays sur les axes factoriels de correspondances multiples

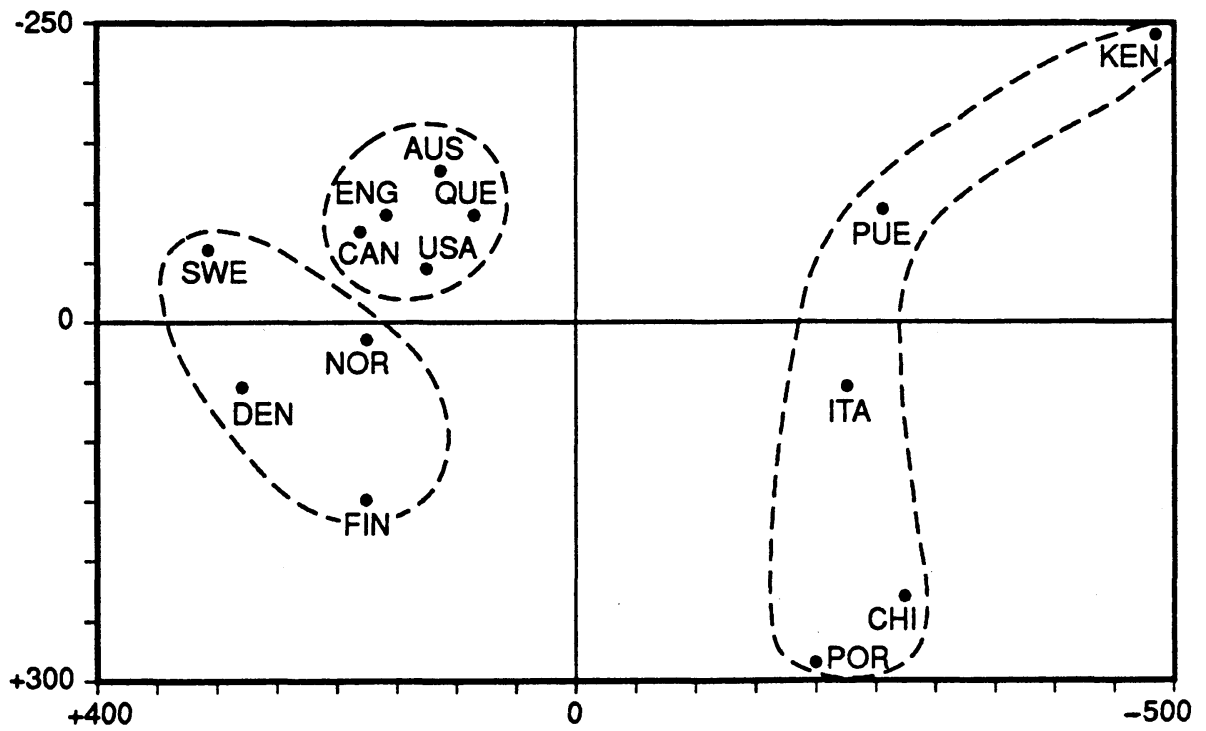

(axe 1 en abscisse et axe 2 en ordonnée)

\subsection{Nouvelles typologies d'entrepreneurs}

Afin de cerner davantage les ressemblances et différences de motivation entre tous ces entrepreneurs, nous avons calculé les fréquences de motivation de $2^{c}$ niveau (voir plus bas) et en avons fait ensuite une analyse CHA, laquelle a révélé sept typologies générales d'entrepreneurs.

Le calcul des fréquences de $2^{c}$ niveau a été fait comme suit :

1. Relevé des variables «discriminantes» de chacun des facteurs ACP (cf. tab.O). Il s'agit des variables de motivation dont la corrélation ( $r^{2}$, «factor loading») est plus élevée que 0,40 .

2. Calcul des fréquences de $2^{\mathfrak{e}}$ niveau pour tous les individus, en utilisant les données recodées et reclassifiées par l'équation personnelle (cf. section 5.4): pourcentages des répondants ayant répondu «très important» ou «important» à chacune des «n» variables discriminantes caractérisant le facteur considéré. Ce calcul est poursuivi pour $(n-1,(n-2)$, etc. jusqu'à $(n-i)$ où $i=n$. Par exemple, le facteur 4 (autonomie, indépendance) dans le tableau 6 est constitué de 11 variables discriminantes, ce qui donne 12 fréquences de $2^{c}$ niveau, alors 
que le facteur 3 (argent) n'est caractérisé que par 3 variables, ce qui produit 4 fréquences. On a ainsi obtenu une matrice de $1926 \times 7$, soit 1926 répondants (ayant répondu à toutes les questions sur la motivation) s'identifiant à chacun des 7 facteurs.

3. Classification des répondants par analyse hiérarchique ascendante des «motivations de $2^{*}$ niveau», i.e. les motivations telles que nous venons de les définir.

Ce demier calcul a révélé l'existence de sept typologies principales d'entrepreneurs dont les principales caractéristiques se résument comme suit :

1. Leur taille est assez semblable : le pourcentage de répondants dans chacun ne varie qu'entre $10 \%$ et $23 \%$.

2. Leur composition est généralement semblable : ces divers groupes se composent d'entrepreneurs de divers pays dans des proportions assez semblables à celles de la population étudiée.

3. Leur identification aux sept facteurs ACP est assez uniforme bien que la typologie 4 soit fortement axée sur l'argent et très peu sur les opportunités, la typologie 1 montre une forte tendance vers l'accomplissement, la typologie 2 penche beaucoup vers l'indépendance et peu vers l'accomplissement, la typologie 3 tend à la fois vers l'accomplissement et l'indépendance.

4. Les trois autres typologies sont «neutres», particulièrement la typologie 5 qui ne se distingue en rien et dont les 250 répondants (13\% de l'échantillon) correspondent de très près à la moyenne mondiale. Toutefois, la typologie 6 se distingue quelque peu en ce sens qu'elle est exceptionnellement faible au niveau de l'accomplissement tout en étant quasi-identique à la typlogie 5 pour le reste. Enfin, la typologie 7 est presque pareille à la typologie 5 sauf qu'elle est «anti-opportunités».

5. Ces diverses typologies affichent à peu près les mêmes caractéristiques de moyennes d'âge, d'éducation et d'antécédents familiaux.

Le manque d'espace nous empêche de présenter ici les résultats détaillés de cette dernière analyse qui ouvre une nouvelle voie pour la compréhension du phénomène entrepreneurial à l'échelle mondiale. Cependant, il n'est sans doute pas trop osé de conclure qu'au-delà des agglomérations régionales, où les entrepreneurs de pays différents se trouvent à partager les mêmes motivations, il existe à travers le monde des individus qui, quels que soient leur pays ou leur culture, sont profondément motivés par des besoins supérieurs, surtout celui de l'accomplissement et du perfectionnement personnel. Pour d'autres, peu nombreux, le besoin primordial est de s'enrichir! 


\section{Discussion}

Bien sûr, ces relations ne disent pas tout. Elles ne sont qu'un éclairage général. En l'absence d'une connaissance approfondie de ces 14 pays, il serait pour le moins présomptueux de notre part de tenter d'expliquer chacune de ces relations. Nous avons plutôt voulu dégager un ensemble général et cohérent, statistiquement valable, de considérations sur les motivations qui animent les entrepreneurs de divers pays.

Les résultats démontrent clairement que la motivation pour fonder une entreprise repose sur plusieurs facteurs dont certains sont psychologiques, d'autres environnementaux ou comportementaux, ou dictés par le besoin d'argent ou la nécessité de se créer un autre espace vital. Les sept principaux facteurs identifiés ont déjà été reconnus dans la littérature mais pas sous l'angle des configurations qui apparaissent ici et dans une optique multiculturelle aussi large. On ne peut pas admettre l'existence d'un simple modèle des motivations des entrepreneurs, comme l'ont suggéré des auteurs comme McClelland (1971).

Nos résultats montrent également que les motivations pour fonder une entreprise ne sont pas arrimées directement aux frontières des pays ou au système culturel qui y prévaut. Au contraire, elles coupent à travers les frontières nationales. Comment expliquer autrement les regroupements obtenus, soit les blocs anglosaxon, scandinave et mixte ? Comment se fait-il que les entrepreneurs britannique, américain, australien, canadien et canadien-français aient les mêmes motivations ? Voilà autant de cultures nationales «contrastées» où les entrepreneurs partagent les mêmes facteurs de motivation qu'avec leurs propres compatriotes. On peut en dire autant des répondants scandinaves. Quant aux répondants du bloc mixte, la conjoncture est moins claire car les effets d'une société communautaire se font sentir. Ces observations mènent au postulat fondamental que la motivation pour créer une entreprise est d'abord influencée par des facteurs métaculturels qui transcendent le pays d'appartenance. Autrement dit, la dynamique et la réceptivité de l'environnement local interviennent beaucoup plus que la culture proprement dite dans la décision de créer une nouvelle entreprise.

D'autre part, peut-on expliquer pourquoi la configuration des Anglo-saxons est dominée par le désir d'indépendance, l'autonomie, l'altruisme, l'évasion et l'accomplissement, alors que celle des Scandinaves est dominée par la recherche d'opportunitéset le rejet du statut social et de l'argent, et la configuration mixte est dominée par l'altruisme, le besoin de reconnaissance sociale et le désir d'avoir de l'argent ?

La configuration des pays anglo-saxons nous renvoie en partie aux valeurs de l'éthique protestante, au calvinisme où l'on accorde une grande importance à l'activité de l'individu, au travail bien fait, à la réalisation, à l'accomplissement et aux droits et devoirs des individus. Les valeurs de l'éthique protestante ne permettent cependant pas d'expliquer l'importance des motivations d'évasion perçues aux ÉtatsUnis, en Angleterre et au Québec. Elles permettent encore moins d'expliquer le 
besoin d'altruisme de l'Australie et de l'Angleterre. Par contre, les besoins d'évasion s'expliquent sans doute par l'industrialisation et la bureaucratisation des firmes.

Dans les sociétés où l'industrialisation a atteint son plein apogée, par exemple en Angleterre et aux États-Unis, on observe que certains individus cherchent à s'éloigner des milieux organisés et verbalisent de la frustration face aux organisations. Ce thème a d'ailleurs alimenté vingt années de recherche sur l'aliénation des travailleurs face à leur milieu.

L'importance que revêtl'altruisme en Australie et en Angleterre est difficile à expliquer.Par contre, la configuration des pays scandinaves est simpleà comprendre en regard de l'orientation politique de ces pays. Depuis plus de 25 ans, ces pays ont mis de l'avant des politiques sociales dont l'objectif est d'offrir à chaque personne des services de base conformément à des valeurs d'égalité et de partage. Il n'est donc passurprenant de voir que les entrepreneurs rejettent les motivations de reconnaissance sociale et d'argent.

La configuration dite mixte est intéressante; elle est dominée par deux motivations de base : reconnaissance sociale et altruisme. Chacun des pays membres de ce groupe accorde une très grande importance àl'une ou l'autre de ces motivations. Comment peut-on expliquer cette configuration? Comment expliquer que dans ces pays, la configuration est habituellement l'inverse de celle que l'on observe dans les pays scandinaves? D'une part, ces pays sont reconnus pour leur collectivisme : ce sont des sociétés communautaires. D'autre part, les études sur les entrepreneurs immigrants ont montré que le désir de s'intégrer à la société, la recherche d'un statut social était une motivation très importante pour ces entrepreneurs. De façon plus spécifique, les études sur les entrepreneurs italiens et chinois (Painchaud et Poulain, 1988; Toulouse et Brenner, 1988) ont démontré que plusieurs d'entre eux créaient une entreprise dont l'objectif était de servir la communauté italienne ou chinoise l'altruisme apparaît alors comme une motivation. Au lieu de parler d'altruisme, certains auteurs ont parlé d'activité d'affaires centrée sur son propre groupe ethnique («ethnocentric entrepreneurial behavior»).

En conclusion, cette étude montre que la motivation des entrepreneurs est primordialement influencée par la nature et la réceptivité de leur environnement. Autrement dit, dans une certaine mesure les entrepreneurs d'un pays ressemblent à ceux de certains autres pays alors qu'au point de vue des valeurs proprement dites, ils ressemblent surtout à leurs compatriotes en général (Blais, 1989).

\section{BIBLIOGRAPHIE}

Alange, S. et Scheinberg, S., (1988), «Swedish entrepreneurship in a cross-cultural perspective», Proc. 1988 Babson Conference on Entrepreneurial Research, Calgary, Alberta, p. 1-14.

Baumol, W.J., (1985), «Entrepreneurship and the long run productivity record», Center for Entrepreneurial Studies, New York University. 
Benzécri, J.-P.etF.,(1989), «Codage linéaire parmorceaux et équation personnelle», Les Cahiers de l'Analyse des Données, vol.14, $\mathrm{n}^{\circ} 3$, p. 331-336.

Blais, R.A., (1989), «Las motivaciones y valores culturales de los empresarios en el mundo», Comptes rendus du $3^{\mathcal{e}}$ Congrès latino-américain sur l'entrepreneurship, Université de Monterrey, Mexico, 25-28 mai, p. 97-115.

Blais, R.A., Blatt, R., Kyle, J.D. et Szonyi, A.J., (1988), «Motivations underlying canadian entrepreneurship in a cross-cultural and cross-occupational perspective», Fifth Canadian Conference of the International Council for Small Business, St. John's, Newfoundland, 14-15 octobre, p. 173-192; Journal of Small Business and Entrepreneurship, (1989), vol. 6, $\mathrm{n}^{\circ} 3$, p. 7-21.

Blais, R.A. et Toulouse, J.-M., (1989), «Motivations pour créer une entreprise», Comptes rendus du $34^{e}$ congrès mondial du Conseil International de la Petite Entreprise, Québec, 21-23 juin, p. 365-378.

Blais, R.A. et Toulouse, J.-M., (1989), «National regional or world patterns of entrepreneurial motivation?», Proc. Sixth Canadian Conference of the International Council for Small Business, University of Windsor, Ontario, 3-4 novembre, p. 50-72.

Blais, R.A., Toulouse, J.-M. et Clément, B., (1990), «International comparisons of entrepreneurial motivation based on personal equation, hierarchical analysis and other statistical methods», Proc. $35 t^{h}$ World Conference of the International Council for Small Business, Washington, D.C., 8-10 juin, p. 2-9.

Blatt, R., Kyle, J.D., Szonyi, A.J. et Blais, R.A., (1989), «The ideology of the canadian entrepreneur», Trans. 34thInt. Council of Small Business, Québec, 21-23 juin, p. 379-393.

Bonacich, E., (1973), «A theory of middleman minorities», American Sociological Review, vol. $38, n^{\circ} .4$, p. $588-594$.

Bruno, A.V. et Tyebjee, T.T., (1982), «The environment for entrepreneurship», dans Encyclopedia of Entrepreneurship, Kent/Sexton/Vesper (Éd.); Englewood Cliffs(N.J.), Prentice-Hall, p. 288-315.

Burgess, S., (1986), The NovaScotiaEthno-Cultural Entrepreneurial Experience, Department of the Secretary of State, Regional Office, Halifax.

Cochran, T., (1960), «Cultural factors in economic growth», Journal of Economic History, vol. 20, p. 515-530.

Cochran, T., (1971), «The entrepreneur in economic change», dans P. Kilby (Ed.), Entrepreneurship and Economic Development, New York, The Free Press.

Collins, O., Moore, D. et Unwalla, D., (1964), The Enterprising Man, Bureau of Business and Economic Research, Michigan State University.

Cooper, A. et Komives, J., (1970), Technical Entrepreneurship, The Center for Venture management, Milwaukee.

Dubini, P., (1989), «The influence of motivations and environment on business startups : some hints for public policies», Journal of Business Venturing, vol. 4, $n^{\circ} 1$, p. 11-26.

Fleming, D., (1988), Creating Entrepreneurs, Sydney, Australie : Allen \& Unwin.

Friberg, M., (1976), «Ar Lonen det enda som sporrar oss att arbeta?» (Is the salary the only incentive for work ?), Sociologisk Forskning, $\mathrm{n}^{\circ} 1$.

Fromm, E., (1941), Escape from Freedom, New York, Rinehart and Winston.

Gasse, Y., (1982), «L'entrepreneur moderne : attributs et fonctions», Revue Internationale de Gestion, vol. $7, \mathrm{n}^{\circ} 4$.

Hagen, E., (1962), On the theory of Social Change, Homewood, Illinois, Dorsey Press.

Hagen, E., «How economic growth change begins : a theory of social change», dans P. Kilby (Éd.), op. cit. 
Hersch, P. et Schiebe, B., (1967), «Reliability and validity of internal-external control as a personality dimension", Journal Consulting Psychology, vol. 31, p. 609-613.

Herzberg, F., Mausner, B. et Snyderman, B., (1959), The Motivation to Work, New York, John Wiley \& Sons.

Hofstede, G., (1980), Culture's Consequences : International Differences in Work Related Values. Beverley Hills, Cal., Sage Publications.

Hornady, J. et Aboud, J., (1971), «Characteristics of successful entrepreneurs», Personnel Psychology, vol. 24, n 2, p. 141-152.

Jacobowitz, A. et Vilder, D., (1982), «Characteristics of entrepreneurs' implication for vocational guidance», Vocational Guidance Quarterly, vol. 30, n 3, p. 252-257.

Kent, C., (1982), «Entrepreneurship education for women. A Research Review and Agenda», Trans. 1982 Meeting of the Joint Council on Economic Education, Washington, D.C.

Knight, R., (1983), «Entrepreneurship in Canada», Journal of Small Business Canada, vol. 1, $\mathrm{n}^{\circ} 1$, p. 9-14.

Kyle, J.D., Blais, R.A., Blatt, R. et Szonyi, A.J., (1989), «The culture of the entrepreneur : fact or fiction», Proc. 1989 Babson Conference of Entrepreneurship Research, St. Louis, Missouri, 27-29 avril.

Lafriente, A. et Salas, W., (1989), «Types of entrepreneurs and firms : the case of new spanish firms», Strategic Management Journal, vol. 10, p. 17-30.

Leff, N., (1979), «Entrepreneurship and economic development : the problem revisited», Journal of Economic Literature, vol. 1, p. 46-64.

Light, I., «Immigrants and ethnic enterprise in North America», Ethnic and Racial Studies, vol. 7, $\mathrm{n}^{\circ} 2$, p. $192-216$.

Lodge, G.C., (1976), The New American Ideology, New York, Alfred A. Knopf.

Maslow, A., (1954), Motivation and Personality, New York, Harper and Row.

McClelland, D.C., (1961), The Achieving Society, Princeton (N.J.), D. Van Nostrand.

McClelland, D.C. et Winter, D., (1969), Motivating Economic Achievement., New York, The Free Press.

McClelland, D.C., (1971), «The achievement motive in economic growth», dans P. Kilby (Éd.), op. cit..

O'Reilly, C.A. et Roberts, K.H., (1973), «Job satisfaction among whites and nonwhites», Journal. of Applied Psychology, vol. 57, n 3, p. 295-299.

Painchaud, C. et Poulin, R., (1988), Les Italiens de Montréal, Montréal, Éditions Asticon.

Pyong Gap, Min, (1984), «From white-collar occupations to small business : korean immigrants occupational adjustment", The Sociological Quarterly, vol. 25, p.333-352.

Pyong Gap, Min, (1987), «Filipino and korean immigrants in small business : a comparative analysis», Amerasia, vol.13, $\mathrm{n}^{\circ} 1, \mathrm{p}$. 53-71.

Richman, C., (1982), Small Business for Workers With Disabilities, Virginia, Institute for Information Studies, Falls Church.

Ronstadt, R., (1984), Entrepreneurship, Dover, Lord Publishing Co.

Rotter, J., (1964), Social Learning and Clinical Psychology, Englewood Cliffs (N.J.), PrenticeHall.

Scheinberg, S. et MacMillan, I.C., (1988), «An 11 country study of motivations to start a business», Frontiers of Entrepreneurial Research, vol. 8, p. 669-687.

Sexton, D. et Bowman, N., (1983), «Determining entrepreneurial potential of students», Proc. US. Academy of Management, p. 408-411.

Shapero, A., (1975), «The displaced uncomfortable entrepreneur», Psychology Today, novembre, p. 83-88. 
Shapero, A. et Sokol, L., (1982), «The social dimensions of entrepreneurship», Chapitre IV, Encyclopedia of Entrepreneurship, Kent/Sexton/Vesper, Editors, Englewood Cliffs(N.J.), Prentice-Hall, p. 72-88.

Stewart, A., (1987), «Entrepreneurs in and at their culture», Working Paper, Brook University, School of Administration Studies.

Toulouse, J.-M., (1979), L'Entrepreneurship au Québec, Montréal, Presses H.E.C./Fides.

Toulouse, J.-M. et Brenner, G.A., (1988), «Les entrepreneurs immigrants : à la recherche d'un modèle théorique», Chaire d'entrepreneurship Maclean Hunter, École des Hautes Études Commerciales, Montréal, Rapport de recherche 88-05-01, mai.

Woodrun, E., (1985), «Religion and economics among Japanese Americans : a weberian study», Social Forms, vol. 64, n 1, p. 191-204. 